\title{
Spectral and Timing Analysis of NuSTAR and Swift/XRT Observations of the X-Ray Transient MAXI J0637-430
}

\author{
Hadar Lazar $^{1}$ (D), John A. Tomsick ${ }^{1}$ (D), Sean N. Pike ${ }^{2}$ (D), Matteo Bachetti ${ }^{3}$ (D), Douglas J. K. Buisson ${ }^{4}$, Riley M. T. Connors ${ }^{2}$ (D),

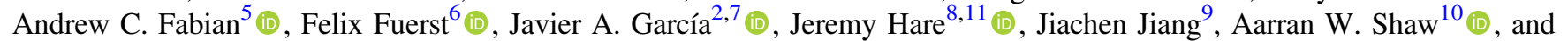 \\ Dominic J. Walton ${ }^{5}$ (i) \\ ${ }^{1}$ Space Sciences Laboratory, 7 Gauss Way, University of California, Berkeley, CA 94720-7450, USA \\ ${ }^{2}$ Cahill Center for Astronomy and Astrophysics, California Institute of Technology, Pasadena, CA 91125, USA \\ ${ }^{3}$ INAF-Osservatorio Astronomico di Cagliari, via della Scienza 5, I-09047 Selargius, Italy \\ ${ }^{4}$ Department of Physics and Astronomy, University of Southampton, Highfield, Southampton SO17 1BJ, UK \\ ${ }^{5}$ Institute of Astronomy, University of Cambridge, Madingley Road, Cambridge CB3 OHA, UK \\ ${ }^{7}$ Dr. Karl Remeis-Observatory and Erlangen Centre for Astroparticle Physics, Sternwartstr. 7, D-96049 Bamberg, Germany \\ ${ }^{8}$ NASA Goddard Space Flight Center, Greenbelt, MD 20771, USA \\ ${ }^{9}$ Department of Astronomy, Tsinghua University, Shuangquing Road 30, Beijing 100084, People's Republic of China \\ ${ }^{10}$ Department of Physics, University of Nevada, Reno, NV 89557, USA \\ Received 2021 June 17; revised 2021 July 28; accepted 2021 August 5; published 2021 November 10
}

\begin{abstract}
We present results for the first observed outburst from the transient X-ray binary source MAXI J0637-430. This study is based on eight observations from the Nuclear Spectroscopic Telescope Array (NuSTAR) and six observations from the Neil Gehrels Swift Observatory X-Ray Telescope (Swift/XRT) collected from 2019 November 19 to 2020 April 26 as the $3-79 \mathrm{keV}$ source flux declined from $8.2 \times 10^{-10}$ to $1.4 \times 10^{-12}$ erg cm $\mathrm{cm}^{-2} \mathrm{~s}^{-1}$. We see the source transition from a soft state with a strong disk-blackbody component to a hard state dominated by a power-law or thermal Comptonization component. NuSTAR provides the first reported coverage of MAXI J0637-430 above $10 \mathrm{keV}$, and these broadband spectra show that a two-component model does not provide an adequate description of the soft-state spectrum. As such, we test whether blackbody emission from the plunging region could explain the excess emission. As an alternative, we test a reflection model that includes a physical Comptonization continuum. Finally, we also test a spectral component based on reflection of a blackbody illumination spectrum, which can be interpreted as a simple approximation to the reflection produced by returning disk radiation due to the bending of light by the strong gravity of the black hole. We discuss the physical implications of each scenario and demonstrate the value of constraining the source distance.
\end{abstract}

Unified Astronomy Thesaurus concepts: Accretion (14); Low-mass X-ray binary stars (939); Black hole physics (159)

\section{Introduction}

A low-mass X-ray binary (LMXB) consists of a compact object, which is either a black hole or neutron star, that accretes matter from a low-mass donor star. As there are fewer than 200 cataloged LMXBs (Liu et al. 2007) in the Galaxy and the Magellanic Clouds, they provide a unique window into the endpoint of stellar evolution. Of particular interest is the interplay between the strong gravity of these elusive compact objects and their respective time-dependent accretion disks. Phase-resolved spectroscopy can be used to understand the processes that occur close to the neutron star surface or the black hole horizon.

The outer region of an accretion disk around the compact object typically cycles between a neutral, quiescent state and an ionized, outburst state. According to the disk instability model (Dubus et al. 2001; Lasota 2001), the matter accumulating on the thin accretion disk heats and ionizes a significant portion of the disk and causes a thermal-viscous instability. The increased viscosity of the disk in an ionized state causes a greater outward redistribution of angular momentum, which is thought to be physically mechanised by the magnetorotational instability (Balbus \& Hawley 1991; Tetarenko et al. 2018). This sharply increases the accretion rate, which in turn causes the

\footnotetext{
${ }^{11}$ NASA Postdoctoral Program Fellow.
}

X-ray luminosity to rise several orders of magnitude above the quiescent state. As the timescale of this bright outburst lasts from days to months, it provides an opportunity for X-ray telescopes to study the poorly understood accretion process.

A typical LMXB has notable spectral features. The accretion disk surrounding the compact object has opacities that are dominated by free-free absorption, and it can be modeled as a disk blackbody (Shakura \& Sunyaev 1973). The soft X-ray photons originating from the standard accretion disk undergo inverse Comptonization on hot electrons in the corona, and produce the hard power-law component (Haardt \& Maraschi 1993; Zdziarski et al. 1993). Thus, the two spectral states can be approximated with (1) a soft thermal multicolored blackbody component and (2) a hard nonthermal power-law component. Additionally, they show intermediate behavior as the sources transition between these states.

Although both types of compact objects may include these components, in neutron stars the presence of a hard surface means that excess energy of accretion is released upon impact and radiated to infinity. This manifests in the neutron star (NS) surface having a high-temperature blackbody component. It is for this reason that black holes are associated with ultrasoft accretion with a lower-temperature thermal component. Generally, the two components that inform this are detectable in soft X-rays $(<10 \mathrm{keV})$. 
The previous analyses in this energy band of the 2019-2020 outburst of black hole candidate (BHC) MAXI J0637-430 (Tetarenko et al. 2020; Jana et al. 2021) demonstrate that the spectral properties are consistent with those of a black hole X-ray binary (BHXRB). Jana et al. (2021) report no signs of highfrequency kilohertz quasi-periodic oscillations, which are seen in several NS LMXBs. Power density spectra (PDSs) are also provided and show that the power decreased rapidly at frequencies $>10 \mathrm{~Hz}$, in accordance with other BHXRBs. Jana et al. also attempted to add an emission component for potential emission from the surface of a neutron star, but found it to be insignificant in their energy band. Though these results favor a black hole as the compact object, they do not rule out a neutron star.

We analyze the broadband spectra of MAXI J0637-430 covering up to $79 \mathrm{keV}$ and report excess emission beyond the two-component model revealed in the soft state. This additional component may be attributed to blackbody emission from the plunging region, i.e., the perceived "boundary" of the black hole itself, which has been identified in a recent study of MAXI J1820+070 (Fabian et al. 2020). This could be modeled with an additional blackbody component associated with the innermost part of the disk and with the start of the plunging region. The reasoning for this is that, while historically models have assumed a zero-stress boundary condition at the innermost stable circular orbit (ISCO) (Novikov \& Thorne 1973; Remillard \& McClintock 2006), later work has suggested that magnetic stresses may occur. These stresses at the ISCO may temporarily delay the plunge of infalling plasma, allowing further energy release and keeping the plasma optically thick to provide additional blackbody flux beyond what is included in standard disk models.

We also consider a reflection spectrum (Fabian et al. 1989) in identifying the extra emission. In many models, the reflected radiation is thought to stem from the reprocessing of high-energy coronal photons in the optically thick accretion disk. The resulting spectrum includes radiative recombination continua, absorption edges, and nuclear emission lines. By modeling the reflection spectrum, one can estimate both the inclination of the accretion disk and the black hole spin parameter by determining the broadening and shape of emission lines.

A common model of this reflection component includes (1) calculation of the reprocessed emission that arises from illumination of the disk by the high-energy Comptonized continuum in the rest frame of the disk and (2) the full raytracing calculations from the irradiating source to the disk and onward to the observer (relxill; Dauser et al. 2014; García et al. 2014). The former feature produces line emissions, notably the iron line emissions, as well as Compton down-scattering of higher energy photons. The latter includes the calculation of the relativistic effects that distort the spectrum, such as light-bending effects, Doppler shifts, and gravitational redshifts. However, some previous studies (e.g., the study of BHXRB XTE J1550 -564; Connors et al. 2020, BHXRB 4U 1630-47 outburst; Connors et al. 2021) have found that the coronal inverseCompton (IC) reflection model does not adequately describe the reflection continuum, and it is necessary to have an alternative model that adopts a softer, thermal continuum as its irradiating spectrum. It was found that the reflection spectrum in the very soft state is best explained by disk self-irradiation, meaning photons from the inner disk are bent by the strong gravity of the black hole and reflected off the disk surface. Despite the theoretical framework of returning radiation being spearheaded decades ago (Cunningham 1976), a full relativistic model for returning thermal radiation reprocessed in the disk has not been produced. Instead, the authors have adopted an approximation to this scenario by implementing reflection models produced with an illuminating blackbody spectrum instead of the standard power-law spectrum typically assumed. This model, called relxillNS (García et al. 2021, submitted), was originally designed to treat reflection in accreting neutron stars, and thus it does not explicitly include the light-bending effects that produce returning radiation. However, it does serve as a proxy of what the reflection signature might look like under these circumstances. Presently, theoretical efforts are underway to produce a selfconsistent model for returning radiation around black holes (e.g., Dauser et al. 2021, submitted).

These components are detectable by telescopes that provide broadband X-ray coverage, sensitivity to the soft emission, and moderate to high spectral resolution. We therefore conducted a broadband X-ray campaign with the Neil Gehrels Swift Observatory X-Ray Telescope (XRT) (Burrows et al. 2005) and the Nuclear Spectroscopic Array (NuSTAR) (Harrison et al. 2013) for the LMXB BHC MAXI J0637-430. Given NuSTAR's ability to simultaneously observe the iron emission line and the Compton hump without pileup effects, it is effective for studying reflection spectra in LMXBs. As NuSTAR's hard X-ray continuum (3-79 keV) allows us to identify the presence of Comptonization, and XRT's soft X-ray continuum (0.5-10 keV) is effective in identifying a disk-blackbody component, these instruments together allow us to identify all the components needed to characterize the LMXB. Moreover, the components can be used to calculate the range of the inner disk radius, which is used to understand the geometry and physics of the LMXB.

When the disk-blackbody component is no longer detected toward the end of the outburst, we turn to characteristic break frequencies obtained from the PDS, because they may also share a relationship with the inner radius of the accretion disk. This is because, as the mass accretion rate drops, the inner radius of a disk $\left(R_{\text {in }}\right)$ increases, causing a drop in the dynamical timescales, and consequently in the characteristic frequencies for the system (Di Matteo \& Psaltis 1999). We can subsequently place an upper bound on the increase in the inner radius from Keplerian orbits, which have the fastest variability timescale at any radius around a compact object. Break frequencies are then compared to changes in the power-law drops in photon index, which previous studies of black hole systems in the hard state have found to be correlated (Gilfanov et al. 1999; Revnivtsev et al. 2001; Kalemci 2002; Tomsick et al. 2004).

The following section outlines the data reduction of the LMXB MAXI J0637-430. Section 3 tests whether the spectra could be adequately described by a model with emission from the plunging region or a model with a reflection component. Section 4 details analysis of the PDS. Lastly, we discuss our results and their physical implications.

\section{Observation and Data Reduction}

MAXI J0637-430 was discovered on 2019 November 2 (MJD 58,789) (Negoro et al. 2019) by MAXI/GSC. Following the discovery, NuSTAR made eight observations from 2019 November 5 to 2020 April 26, six of which were made contemporaneously with XRT observations. This six-month multiwavelength campaign ended when XRT made a nondetection in the X-ray band on 2020 June 11 (Tomsick \& Lazar 2020). In Table 1, we detail the exposure times and source flux evolution of this multiwavelength campaign 
Table 1

Observations

\begin{tabular}{|c|c|c|c|c|c|c|c|}
\hline MJD & $\begin{array}{l}\text { NuSTAR } \\
\text { ObsID }\end{array}$ & $\begin{array}{l}\text { NuSTAR Flux }(3-79 \mathrm{keV}) \\
\quad\left(10^{-10} \mathrm{erg} \mathrm{cm}^{-2} \mathrm{~s}^{-1}\right)\end{array}$ & $\begin{array}{c}\text { NuSTAR Exposure } \\
\text { Time (s) }\end{array}$ & $\begin{array}{l}\text { XRT } \\
\text { ObsID }\end{array}$ & $\begin{array}{l}\text { XRT Flux }(0.5-10 \mathrm{keV}) \\
\left(10^{-10} \mathrm{erg} \mathrm{cm}^{-2} \mathrm{~s}^{-1}\right)\end{array}$ & $\begin{array}{l}\text { XRT Exposure } \\
\text { Time (s) }\end{array}$ & $\begin{array}{c}\text { Model }^{\mathrm{a}} \\
\text { Fit }\end{array}$ \\
\hline 58,792 & 80502324002 & $8.19 \pm 0.02$ & 36,799 & $\ldots$ & $\ldots$ & $\ldots$ & 1 \\
\hline 58,801 & 80502324004 & $6.44 \pm 0.02$ & 67,738 & 00012172008 & $42.23 \pm 0.17$ & 2525 & 1 \\
\hline 58,866 & 80502324008 & $2.37 \pm 0.02$ & 46,630 & 00012172066 & $2.98 \pm 0.07$ & 674 & 2 \\
\hline 58,879 & 80502324010 & $0.72 \pm 0.01$ & 110,779 & 00012172077 & $0.46 \pm 0.02$ & 1686 & 3 \\
\hline 58,889 & 80502324012 & $0.37 \pm 0.01$ & 50,233 & 00012172085 & $0.17 \pm 0.01$ & 1860 & 3 \\
\hline
\end{tabular}

Note.

a (1) Soft state: TBabs x (diskbb + relxillNs + Nthcomp). (2) Transition: TBabs x (diskbb + Nthcomp) . (3) Hard state: TBabs x Nthcomp.

through the soft, intermediate, and hard states. In Figure 1, we provide the XRT light curve for the outburst.

\subsection{NUSTAR}

All NuSTAR data were processed using calibration database (CALDB) files from 2019 September 10 and analyzed using XSPEC version 12.11.1c (Arnaud 1996). We used the nupipeline tool (version v0.4.6) to filter the event lists, and then the nuproducts tool to extract spectra and response files for the focal plane modules A and B (FPMA and FPMB). For the first six observations, the source and background extraction region was a circle of radius $86^{\prime \prime}$. By the last two observations the outburst dimmed, so the radii were reduced to $32^{\prime \prime}$ for 80502324014 and 27 " for 80502324016 . The FPMA and FPMB spectral data were generated as two different groups using GRPPHA in HEASOFT version $6.27 .2^{12}$ with a signal-to-noise ratio of 10 in each bin for the first five observations, a signal-to-noise ratio of 5 for 80502324012 and 80502324014 , and a signal-to-noise ratio of 4 for 80502324016 . An XRT observation was added as a third group when the observations coincided. These groups were then jointly fit using models available in XSPEC and the relxill suite, which provides self-consistent relativistic disk reflection models (Dauser et al. 2014; García et al. 2014). We also used relxillNS, a recent development of the relxill suite, which gives the reflection of the disk illuminated by the neutron star surface or black hole boundary layer. It assumes a blackbody is irradiating the disk, rather than the power law given by the traditional relxill model.

\subsection{Swift/XRT}

We extracted $0.5-10 \mathrm{keV}$ energy spectra for the six Swift/ XRT observations that occurred during NuSTAR observations (Table 1). These observations have relatively short exposure times, covering only a fraction of the much longer NuSTAR observations. We extracted the spectra using Swift data analysis tools in HEASOFT v6.27.2 with the use of CALDB files. The spectra were grouped using a signal-to-noise ratio of 5 in each bin for all observations except the last one of 00012172093, which had a signal-to-noise ratio of 2 . For all of the reductions described below, we only used events flagged with grade 0 .

The XRT count rate changed significantly from 175 counts $\mathrm{s}^{-1}$ in the first observation to 0.05 counts $\mathrm{s}^{-1}$ in the last one. Thus, different XRT instrument modes were used as well as different

\footnotetext{
12 https://heasarc.gsfc.nasa.gov/docs/heasarc/caldb/caldb_wotsnew. html\#nustar_20210825
}

extraction methods for the spectra. For the first four observations, XRT was in windowed timing (WT) mode, and for the first two observations (ObsIDs 00012172008 and 00012172018), the count rate was high enough for photon pileup to be a concern. Thus, for these two observations, the source extraction region was an annulus with inner radius of $20^{\prime \prime}$ and outer radius of $47^{\prime \prime}$. A background spectrum was also extracted from an active detector region far from the source, and the background level was scaled based on the relative sizes of the source and background regions. We used the appropriate response file (swxwt0s6_20131212v015. $\mathrm{rmf}$ ) and used XRTmkarf to produce the ancillary response file. For the other two WT observations (ObsIDs 00012172066 and 00012172077), the count rates are lower, and the source extraction region was a circle of radius $47^{\prime \prime}$ rather than an annulus.

XRT was in photon counting mode for the final two Swift/ XRT observations and the count rates were 0.28 counts $\mathrm{s}^{-1}$ and 0.05 counts $\mathrm{s}^{-1}$. At these low count rates, photon pileup is not a concern, and the extraction region we used was a circle of radius $47^{\prime \prime}$. For these spectra, the appropriate response file is swxpc0s6_20130101v014.rmf, which is the file we used. For all six observations, we used an exposure map when making the ancillary response file. It is important to do this in cases where there are bad pixels or pixel rows in the source region. We especially point this out here because the low count rate for the final observation (ObsID 00012172093) was partly due to the fact that the source was on a row of bad pixels.

\section{Spectral Results}

We carried out spectral studies of the BHC MAXI J0637-430 during its 2019-2020 X-ray outburst using observations from NuSTAR in the 3-79 keV range and observations from XRT in the $0.5-10 \mathrm{keV}$ range. For the joint fitting between XRT, FPMA, and FPMB, a cross-normalization constant is allowed to vary freely with the convention that FPMA is unity. The value of the cross-normalization factor between FPMA and FPMB is found to always lie within NuSTAR's accepted limit of $\leqslant 5 \%$ (Madsen et al. 2015). The neutral hydrogen column density $\left(N_{\mathrm{H}}\right)$ is fixed to $4.39 \times 10^{20} \mathrm{~cm}^{-2}$ (Tetarenko et al. 2020), which was given by the reddening $E(B-V) \sim 0.064$ in Strader et al. (2019), for all the described models. It is noteworthy that relaxing this constraint on the column density appeases low-energy residuals (see the Appendix for more details). All parameter uncertainties are reported at the $90 \%$ confidence level for one parameter of interest. Furthermore, all models described include the Galactic absorption through the implementation of the TBabs model (Wilms et al. 2000). 


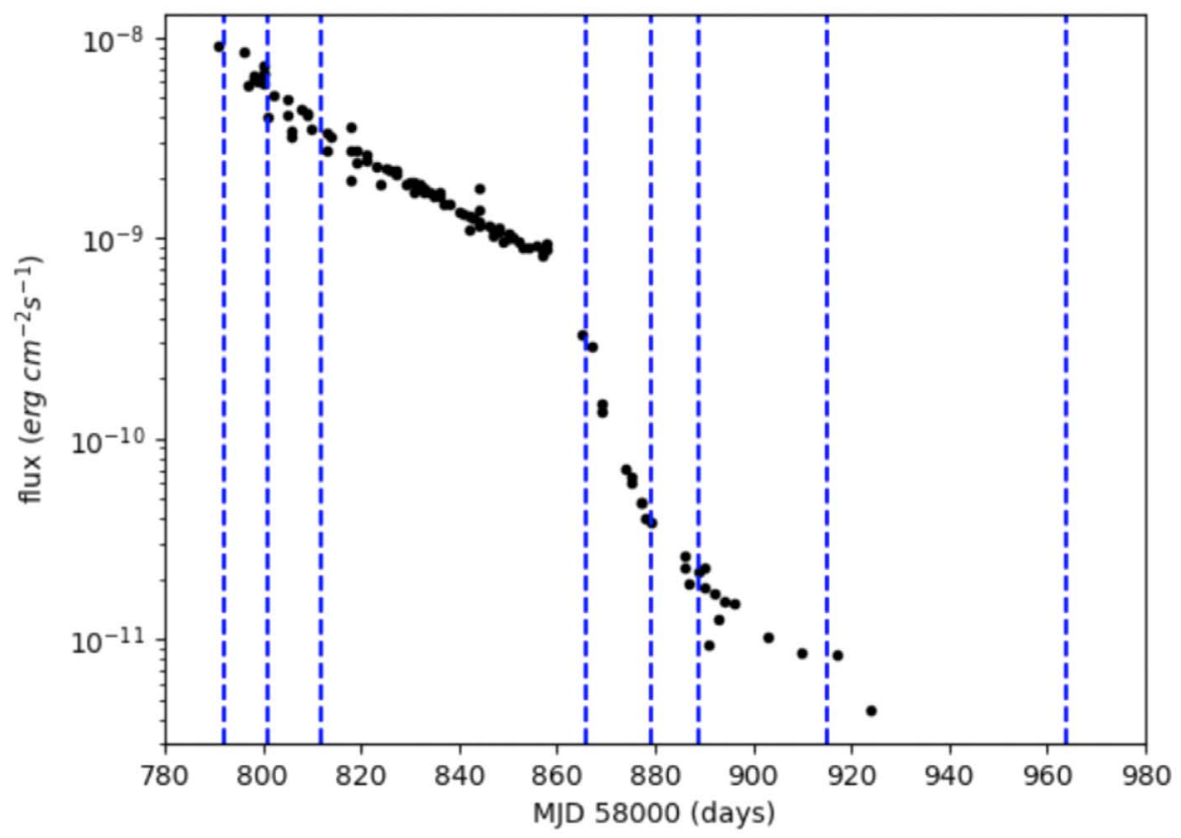

Figure 1. Swift/XRT fluxes for MAXI J0637-430 from Tetarenko et al. (2020). The dashed lines indicate the dates NuSTAR observations were made.

The following two subsections report on tests conducted to determine which model best describes the soft-state spectra. We evaluated the quality of the different models by comparing their $\chi^{2}$ values when fit to the robust observations made on MJD 58,801 and 58,812. The results obtained from observations made on MJD 58,812 are provided below.

\subsection{Soft-state Spectral Results without Reflection}

We first fit the soft-state spectra (see Table 1 for state characterization of each observation) with an absorbed thermal Comptonization model (Zdziarski et al. 1996), TBabs x Nthcomp in XSPEC notation. The Nthcomp component describes the continuum shape from the thermal Comptonization of photons by a cloud of electrons (the "corona"). The presence of positive residuals in soft X-ray ranges, specifically in the rising phase of the outburst, motivated us to add a multicolor blackbody component (Mitsuda et al. 1984; Makishima et al. 1986) to account for the direct photons from the accretion disk. This two-component model, comprising a combination of multicolor disk-blackbody and thermal Comptonization component, TBabs x (diskbb + Nthcomp), provides a better explanation of the observed broadband energy spectra. As the Nthcomp component accounts for the lowenergy rollover due to the seed photons from the accretion disk, the Nthcomp seed photon temperature and the diskbb inner disk temperature were tied together $\left(k T_{\text {in }}\right)$. We note that of the three observations collected for the soft state, the one occurring on MJD 58,792 did not have a simultaneous XRT observation. We therefore omit the diskbb parameter values for this observation, because without the features in the XRT energy range this component is not well constrained.

It is notable that an X-ray campaign recording up to the $10 \mathrm{keV}$ X-ray range reported that the XRT data are well described by two-component model of a disk blackbody and absorbed power law (Tetarenko et al. 2020). However, we find that once we incorporate the higher energy range of NuSTAR, a two-component model does not sufficiently describe the softstate observations, giving a $\chi^{2}$ of 1242 for 720 degrees of freedom (dof) for TBabs x (diskbb + Nthcomp). In particular, we see positive residuals near $6 \mathrm{keV}$, below $1 \mathrm{keV}$, and notably at the higher energies (see Figure 2). We therefore tested whether (i) blackbody emission from the plunging region, (ii) reflection of the high-energy component, or (iii) reflection of a thermal returning radiation component, or a combination of these, provides the best explanation of these spectra.

First, we tested the addition of a single-temperature blackbody component, TBabs x (diskbb + Nthcomp + bbodyrad). This introduces an extra blackbody component associated with the boundary of the black hole, interpreted as the start of the plunging region. This is motivated by the fact that recent work (Fabian et al. 2020) suggests that plasma may remain optically thick slightly within the ISCO, powering the additional emission. When a model accounting for this emission is fitted, we obtain a $\chi^{2}$ of 859 for 718 dof (see Figure 3).

To check for physical consistency of the scenario of a plunging region, we can use the normalization of the blackbody in order to estimate the width of the ring at the ISCO and determine whether it is a physically plausible value. In order to proceed, we make the reasonable assumption that the disk extends to the ISCO, giving us $R_{\mathrm{ISCO}}$ between 1 and $6 R_{\mathrm{g}}$, in which $R_{\mathrm{g}}$ is the gravitational radius. This is consistent with the fact that the model of a plunging region is thought to be most relevant for low-spin black holes, which have $R_{\mathrm{ISCO}}$ close to 6 $R_{\mathrm{g}}$. Using $R_{\mathrm{g}}=G M / c^{2}$ and taking a $\mathrm{BH}$ mass of $10 M_{\odot}$, we find that $R_{\text {ISCO }}$ falls between 15 and $90 \mathrm{~km}$. The normalization of the bbodyrad component is $N_{\text {bbodyrad }}=R_{\mathrm{km}}^{2} / D_{10}^{2}$ Here, $R_{\mathrm{km}}$ is the source radius in kilometers and $D_{10}$ is the distance to the source, and it assumes a spherical geometry of the blackbody. Taking the normalization of $8.6_{-2.6}^{+4.0}$ for MJD 58,812 , and assuming that the source is between 6.5 and $10 \mathrm{kpc}$ away (Jana et al. 2021), we can approximate a range for $R_{\mathrm{km}}$ to estimate a spherical surface area of heat propagation. Assuming that the plunging region is a thin ring at the ISCO, we collapse this spherical surface onto a ring, and find that the 


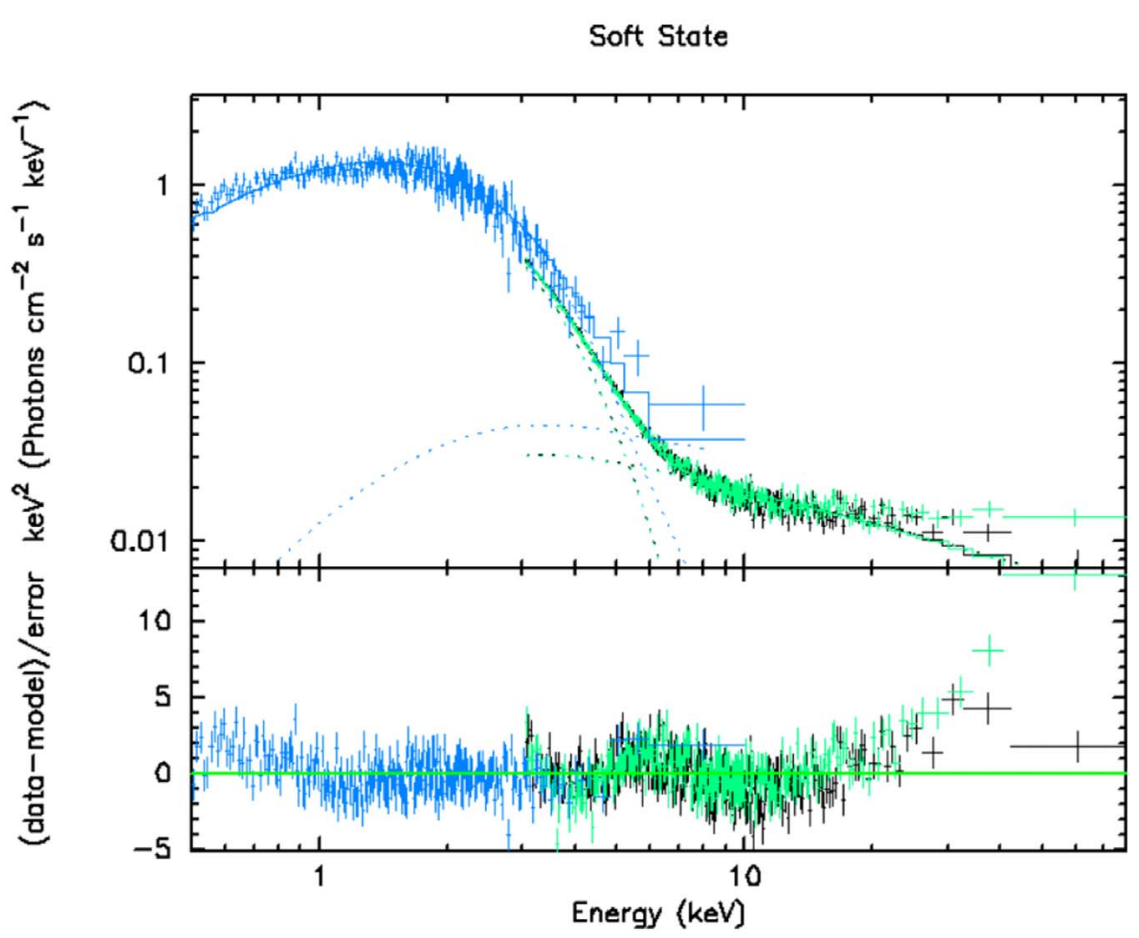

Figure 2. Spectra for NuSTAR"s 80502324006 observation (black for FPMA, green for FPMB) and XRT's 00012172018 observation (blue) fitted with a disk blackbody and a thermal Comptonization component. The fit produces positive residuals at the highest energies, near $6 \mathrm{keV}$, and below $1 \mathrm{keV}$.

width of the ring $\Delta R$ may vary between 0.76 and $1.56 \mathrm{~km}$. As this a physically plausible range for the ring width, we do not rule out the possibility of an extra emission component in the plunging region.

However, the residuals near $6 \mathrm{keV}$ of the fitted spectra are still prominent. In the search for a physically sound model with a better fit, we invoke reflection of the blackbody returning radiation component.

\subsection{Soft-state Spectral Results with Reflection}

This analysis uses relativistic disk reflection models from the relxill model suite. We assume a lamppost geometry of the Comptonizing corona and use the relativistic reflection model in addition to a thermal Comptonization component. The photon temperature from the additional diskbb component is used as the seed temperature for the internal Nthcomp continuum. Here we consider two alternative reflection models for the outburst, one standard and the other with returning radiation. For both models, we found that the spin is unconstrained, and we therefore fixed the spin $a$ to zero. Also, as the relxillns model does not interpolate below an iron abundance value of 0.5 solar, we set that as the lower limit.

At first, we added a reflection component relxillcp, which has an explicit Nthcomp component to represent the continuum, TBabs $\mathrm{x}$ (diskbb + relxillCp). This mitigated the residuals seen for TBabs $x$ (diskbb + Nthcomp) at higher energies, yielding a $\chi^{2}$ of 994 for 717 dof (see Figure 4). This model combines a coronal IC spectrum atop the accretion disk and the full ray-tracing calculations from the irradiating source to the disk and onward to the observer, as discussed in the introduction. The coronal IC spectrum of the relxillCp model is power-law-like, but the early spectra of MAXI J0637-430 are dominated by a softer, blackbody-like continuum.
We therefore employ the model relxillns, which computes illumination of the disk by a blackbody spectrum instead of the cutoff power law of the original relxill model or the extra Comptonization component of relxillcp. The model finds values for the blackbody temperature $k T_{\mathrm{bb}}$, log of the ionization parameter, iron abundance $A_{\mathrm{Fe}}, \log$ of the density of the disk, and inclination. It is necessary to set a lower limit on the ionization parameter $\xi=L / n R^{2}$ where $L$ is the X-ray luminosity, $n$ is the density, and $R$ is the distance from the $\mathrm{X}$-ray source to the illuminated material. Taking the distance from the source to be 6.5 to $10 \mathrm{kpc}$, we convert the fluxes in the soft state (see Table 1) to luminosities and find that they are of the order of $10^{37} \mathrm{erg} \mathrm{s}^{-1}$. We then take the density to be $n=1 \times 10^{19} \mathrm{~cm}^{-3}$ and the distance from the source to the illuminated material to be between $10^{7}$ and $10^{8} \mathrm{~cm}$. We obtain a lower limit of $\log \xi>3$ for the ionization parameter.

Figure 5(a) displays the last model analyzed, which includes components for a disk blackbody, thermal Comptonization, and reflection of blackbody returning radiation (TBabs $x$ (diskbb + relxillns + Nthcomp) ). It provides a fit with a $\chi^{2}$ of 832 for 713 dof (see Table 2). Figure 5(b) applies the new fit to the same soft-state observation as Figure 2, in order to demonstrate the improvement. For a detailed breakdown of the parameters of this soft state model of our most data-rich observations, see Table 3 .

We constrained the distance of the compact object by equating $R_{\text {in }}$, the inner edge of the disk, with the ISCO. The normalization of diskbb is given by $N_{\text {disbb }}=\left(r_{\text {in }} / D_{10}\right)^{2} \cos \theta$. Here, $r_{\text {in }}$ is the apparent disk radius in kilometers, $D_{10}$ is the source distance in units of $10 \mathrm{kpc}$, and $\theta$ is the inclination angle. Our relationship between the inner edge of the disk and the apparent edge is given by $R_{\text {in }}=\epsilon \kappa^{2} r_{\text {in }}$, in which $\epsilon=0.41$ is a factor (Kubota et al. 1998) correcting the gross multicolored disk formalism to the inner boundary condition, and $\kappa=1.7-2.0$ is the spectral hardening factor (Shimura \& Takahara 1995). Taking $\kappa=1.8$, we use the 

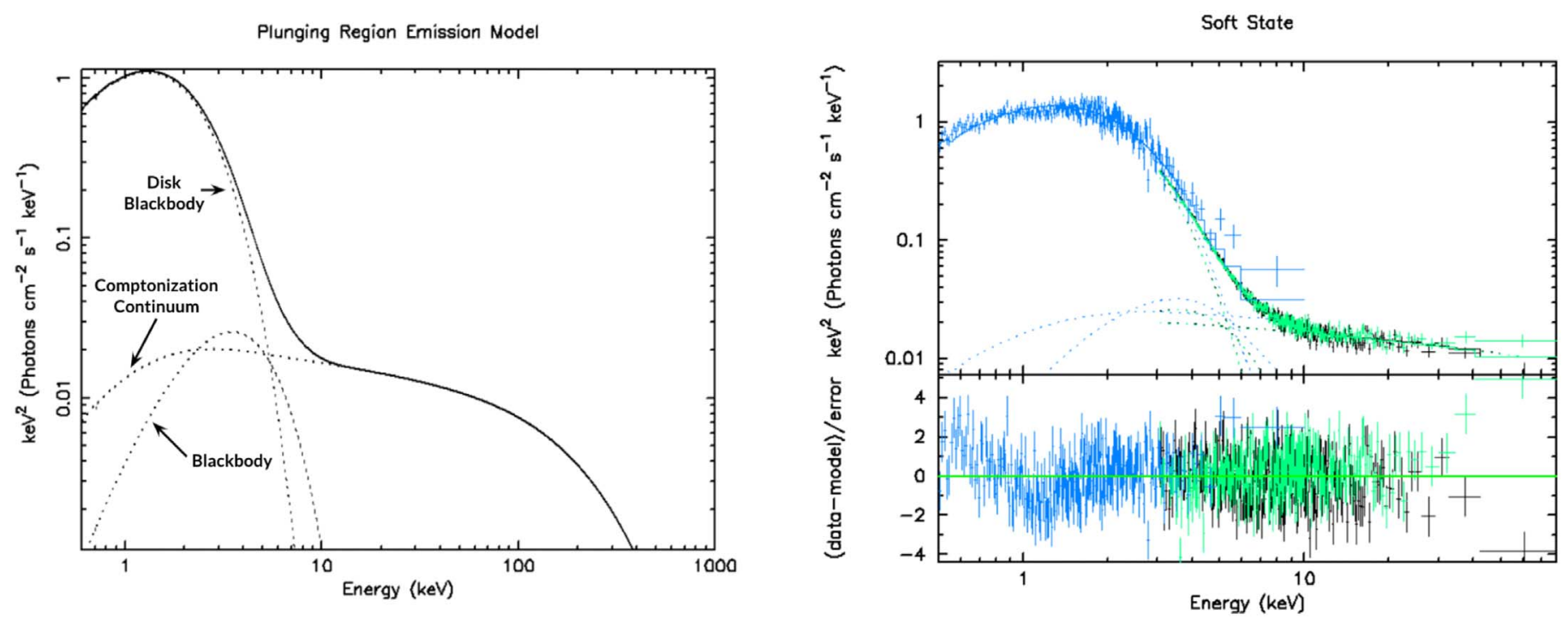

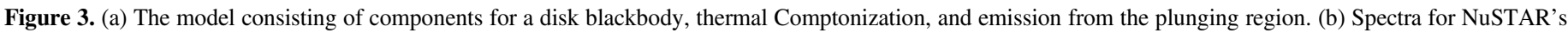

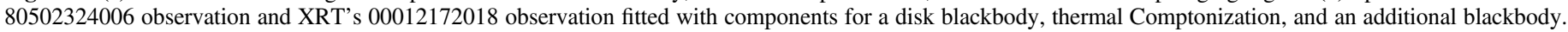

diskbb normalization to find that the inner radii for the observations made on MJD 58,801 and 58,812 are $R_{\text {in }}=$ $\left(84_{-26}^{+27}\right) D_{10}(\cos \theta)^{-1 / 2} \mathrm{~km}$ and $R_{\text {in }}=\left(77_{-26}^{+24}\right) D_{10}(\cos \theta)^{-1 / 2} \mathrm{~km}$ (see Table 4).

If the compact object may be a nonrotating black hole, we can use the mean inner radius to ultimately find the mass of the $\mathrm{BH}$. We therefore have

$$
R_{\text {in }}=(81 \pm 26) D_{10}(\cos \theta)^{-1 / 2} \mathrm{~km}
$$

For a Schwarzschild black hole, $R_{\mathrm{ISCO}}=6 R_{\mathrm{g}}$, giving

$$
M=(9 \pm 3) D_{10}(\cos \theta)^{-1 / 2} M_{\odot} \text {. }
$$

This constraint on $R_{\mathrm{in}}$ and $M$ is consistent with results provided by a NICER and XRT study of MAXI J0637-430 outburst in the $0.5-10 \mathrm{keV}$ energy range (Jana et al. 2021).

An advantage of the additional higher energy band that NuSTAR provides is that it allows us to detect a reflection component in the soft state, which, as discussed, gives an estimation for the inclination angle. The mean inclination angle in the soft state found in this study is $64^{\circ} \pm 6^{\circ}$, which yields

$$
M=(14 \pm 6) D_{10} M_{\odot} .
$$

The distance estimate of $6.5-10 \mathrm{kpc}$ leads to a black hole mass similar to the masses found in other black hole binaries. A similar re-derived distance estimate for this analysis is found in the discussion, as well as a distance estimate for a spinning black hole. As this model gives the most optimal fits and results in plausible estimates for the inner edge of the disk and mass of the $\mathrm{BH}$, we conclude that the soft state of MAXI J0637-430 is well described by it. Table 2 provides a summary of the reduced $\chi^{2}$ values of the different models tested in the soft state, with a model including a relxillNS component being the best fit for both observations.

\subsection{Spectral Results through State Transition}

Throughout our observations of MAXI J0637-430, we see the source transition from a soft state with a strong diskblackbody component to a hard state dominated by a thermal Comptonization component. Figure 6 shows the outburst through state transition, in which the diskbb component is still quite strong, with an inner disk temperature that fell by a factor of three. Finally, in Figure 7, we show that the disk component is not detectable in the hard state.

We can use the normalization of diskbb once again to obtain the inner edge radius $R_{\text {in }}$ of the disk in the soft-hard state on MJD 58,866, which results in

$$
R_{\text {in }}=\left(229_{-116}^{+102}\right) D_{10}(\cos \theta)^{-1 / 2} \mathrm{~km} .
$$

This shows that when blackbody reflection is no longer detected in this intermediate state, the range of $R_{\text {in }}$ increases by a factor of three while temperature drops by a factor of three (see Table 4). The drop in inner disk temperature and increase in $R_{\text {in }}$ in the soft-to-hard transition indicate that softer reflected emission due to self-irradiation is associated with a hotter disk with a smaller inner radius. The spectral results for an outburst of the black hole binary (BHB) 4U 1630-47 (Connors et al. 2021) also found this association for the soft state.

The defining models used are TBabs $\mathrm{x}$ (diskbb + relxillNs + Nthcomp) in the soft state, TBabs $x$ (diskbb + Nthcomp) in the transitional state, and TBabs $x$ Nthcomp in the hard state. Table 1 indicates which observations correspond to each model. The state is seen to transition on MJD 58,866, when the reflection component in the NuSTAR energy range is no longer detected. The parameter values throughout the observations are summarized in Table 4 and Figure 8.

In the hard state, we no longer detect a disk-blackbody component. We can use the cross power density spectrum (CPDS) to obtain break frequencies, which may trace the inner radius even after the thermal component falls below our bandpass. The following section details this procedure.

\section{Analyzing the Cospectra}

Rather than studying the PDS for each observation, we utilized the fact that NuSTAR observes simultaneously with two instruments, FPMA and FPMB, in order to produce the 

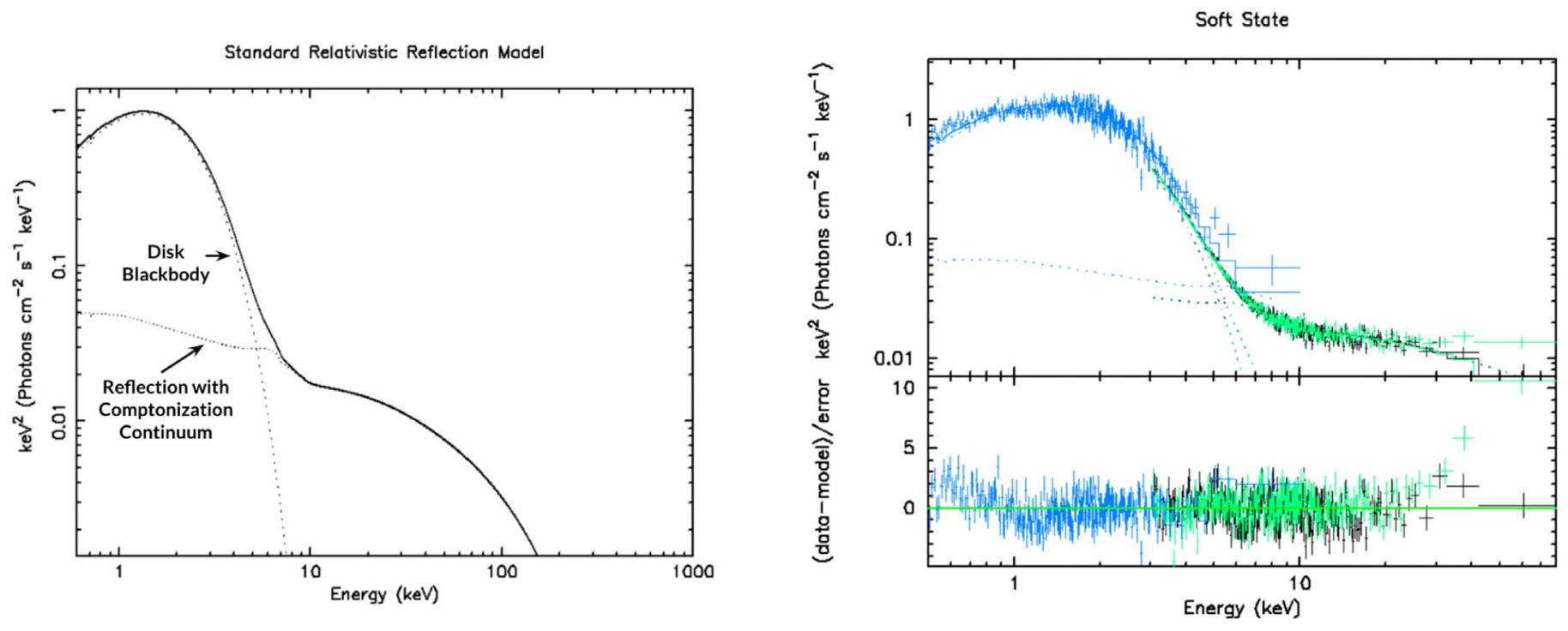

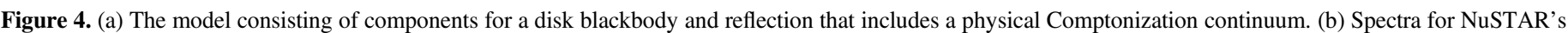

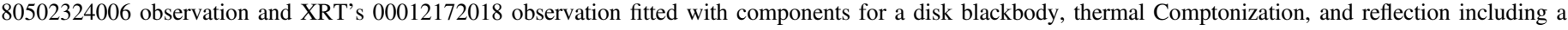
physical Comptonization continuum.

CPDS for each observation. The CPDS is given by

$$
C(\nu)=\mathcal{F}_{\mathrm{A}}^{*}(\nu) \mathcal{F}_{\mathrm{B}}(\nu)
$$

where $\mathcal{F}_{\mathrm{A}}^{*}(\nu)$ is the complex conjugate of the Fourier transform of the light curve observed by FPMA and $\mathcal{F}_{\mathrm{B}}(\nu)$ is the Fourier transform corresponding to FPMB. The principal advantage of the CPDS over the PDS is the fact that its real part, the cospectrum, only includes the power due to signals that are in phase between the two light curves, thereby eliminating contributions due to background and dead time (Bachetti et al. 2015).

We began by shifting the arrival times in the cleaned event files to the barycenter of the solar system by calling barycorr while extracting scientific products with nuproducts. Calling barycorr in this way defaults to the FK5 reference frame and the corresponding ephemeris DE-200. We specified the coordinates of the source in the FK5 reference frame as determined by the automatic centroid detection tool provided by DS9. In order to avoid introducing an artificial offset between FPMA and FPMB arrival times, we used the source coordinates as determined using FPMA to correct the event files for both modules. We supplied the NuSTAR clock correction file v111 generated on 2020 October 30. Using the Stingray package (Huppenkothen et al. 2019), we then produced FPMA and FPMB light curves with resolution 1/ $512 \mathrm{~s}$, filtering events using source regions with radius $90^{\prime \prime}$ and centered on the source using the automatic centroid function provided by DS9. We did not filter for energy, instead producing light curves for the entire NuSTAR energy range (3-79 keV). Next, we split the light curves into segments of length $256 \mathrm{~s}$. For each pair of segments, corresponding to FPMA and FPMB, we produced the cospectrum. All of the cospectra produced for a given observation were then averaged, resulting in the average cospectra shown in Figure 9, which have been rebinned for clarity.

The cospectra appear relatively featureless, aside from lowfrequency noise. In order to characterize the shape of this noise, we fit each cospectrum with a single zero-centered Lorentzian, described by

$$
P(\nu)=\frac{A}{\pi}\left[\frac{\gamma}{\nu^{2}+\gamma^{2}}\right]
$$

where $P(\nu)$ is the rms-normalized power density, $A$ is the total integrated power under the Lorentzian, and $\gamma$ is the half-width at half-maximum. The total power, accounting only for positive frequencies, is given by $A / 2$. This is related to the total $\mathrm{rms}$ of the component (in units of percent) by the relation $\mathrm{rms}=$ $\sqrt{A / 2} \times 100$.

Figure 8 and Table 4 summarize the evolution of the values derived from power spectra. We note that throughout the outburst, $R_{\text {in }}$ increases and the half-width frequency decreases. Meanwhile, the power-law photon index $(\Gamma)$ drops. The implications of this are further explored in the discussion.

\section{Discussion}

\subsection{Evolution of Spectral and Timing Properties over Time}

This analysis classified MAXI J0637-430 in three spectral states: soft, intermediate, and hard. To detail the change of properties through state transition, we provide the results of the spectral and timing analysis over time in Figure 8, showing the evolution of (a) the source flux for XRT (Tetarenko et al. 2020) and NuSTAR, (b) the power-law photon index, (c) the electron temperature of the corona in $\mathrm{keV}$, (d) the inner disk temperature in $\mathrm{keV}$, (e) the rms (\%) derived from the power spectra, and (f) break frequencies from power spectra.

The light curve in Figure 8(a) displays the flux changes in XRT's $0.5-10 \mathrm{keV}$ energy band and NuSTAR's 3-79 keV band. NuSTAR's hard X-ray continuum is advantageous in identifying the presence of Comptonization, while XRT's soft $\mathrm{X}$-ray continuum helps to identify a disk-blackbody component. Consequently, XRT observes higher fluxes in early softstate observations than NuSTAR. The shift in dominance to NuSTAR in later observations illustrates the transition to the hard state. The NICER campaign (Jana et al. 2021) divided the transitions into finer increments of the soft-intermediate state, 


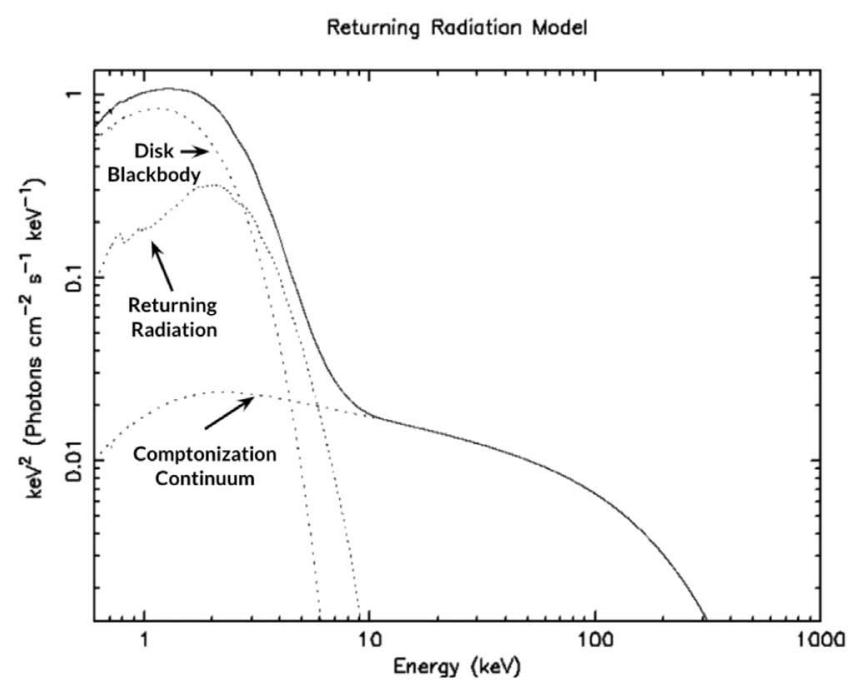

(a)

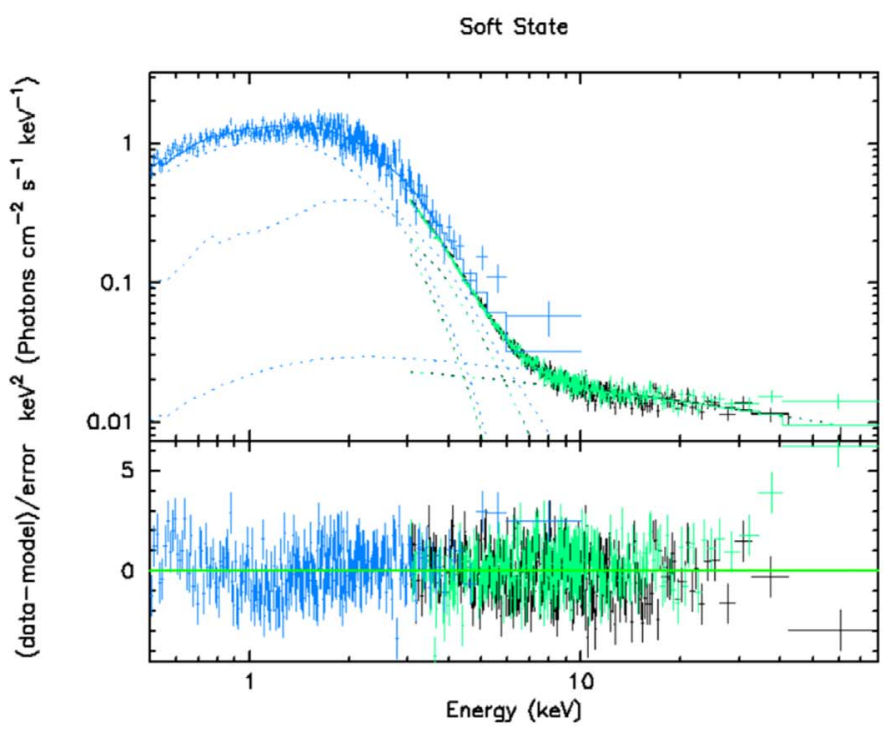

(b)

Figure 5. (a) The model consisting of components for a disk blackbody, thermal Comptonization, and reflection of blackbody returning radiation. (b) Spectra for NuSTAR's 80502324006 observation and XRT's 00012172018 observation fitted with components for a disk blackbody, thermal Comptonization, and reflection of blackbody returning radiation.

Table 2

Testing Soft-state Fits

\begin{tabular}{lcc}
\hline \hline Model & $\begin{array}{c}\chi^{2} / \text { dof } \\
\text { MJD 58,801 }\end{array}$ & $\begin{array}{c}\chi^{2} / \text { dof } \\
\text { MJD 58,812 }\end{array}$ \\
\hline TBabs x (diskbb + Nthcomp) & $2374 / 1158$ & $1242 / 720$ \\
TBabs x (diskbb + Nthcomp + & $2154 / 1156$ & $859 / 718$ \\
bbodyrad) & & \\
TBabs x (diskbb + relxillCp) & $1901 / 1155$ & $944 / 717$ \\
TBabs x (diskbb + relxillNs + & $1320 / 1151$ & $832 / 713$ \\
Nthcomp) & & \\
\hline
\end{tabular}

high soft state, hard-intermediate state (HIMS), and low hard state by tracking the photon power-law index and the correlation between spectral states and timing properties. We did not distinguish between the two flavors of the soft state because we did not obtain fitted power-law photon indices indicating a transition. As for later states, our intermediate-state observation occurred when Jana et al. (2021) indicate that the source was in the HIMS.

A combination of the spectral analysis in the soft and intermediate states and timing analysis in the hard state makes it possible to map out the evolution of the accretion disk geometry throughout the outburst. The disk-blackbody component in the soft and intermediate states (Sections 3.2 and 3.3) indicates that the range of the inner disk radius increases by a factor of three. Once the source is in the hard state and the diskblackbody component is no longer detected, we use the properties of the power spectrum to constrain the inner radius following Di Matteo \& Psaltis (1999). We therefore then use the Lorentzian half-width frequency to trace the inner disk radius once the disk-blackbody component is no longer detectable in the spectra. The Lorentzian half-width frequency is found to decrease from the intermediate state to the hard state by a factor of 21 . We can place an upper bound on the increase in the inner radius from Keplerian orbits using $R \propto \nu^{-2 / 3}$, where $\nu$ is the characteristic frequency. Therefore, the decrease
Table 3

Soft-state Model ${ }^{\mathrm{a}}$

\begin{tabular}{lccc}
\hline \hline Model & Parameter & MJD 58,801 & MJD 58,812 \\
\hline TBABS & $N_{\mathrm{H}}\left(10^{22} \mathrm{~cm}^{-2}\right)$ & $4.39 \times 10^{-2}$ & $4.39 \times 10^{-2} \dagger^{\mathrm{b}}$ \\
DISKBB & $k T_{\text {in }}(\mathrm{keV})$ & $0.45_{-0.03}^{+0.02}$ & $0.44_{-0.01}^{+0.02}$ \\
& norm & $3964_{-378}^{+410}$ & $3312_{-384}^{+319}$ \\
RELXILLNS & $i(\mathrm{deg})^{\mathrm{c}}$ & $70_{-1}$ & $58_{-7}^{+7}$ \\
& $k T_{\mathrm{bb}}(\mathrm{keV})$ & $0.523_{-0.002}^{+0.003}$ & $0.529_{-0.003}^{+0.010}$ \\
& $\log \xi^{\mathrm{d}}$ & $3^{+0.01}$ & $3^{+0.01}$ \\
& $A_{\mathrm{Fe}}$ & $0.5^{+0.04}$ & $0.5^{+0.06}$ \\
& $\log N$ & $17.3_{-0.3}^{+0.4}$ & $17.3_{-0.2}^{+0.6}$ \\
NTHCOMP & $\operatorname{norm}\left(10^{-5}\right)$ & $21_{-0.3}^{+8}$ & $8_{-1}^{+4}$ \\
& $\Gamma$ & $2.06_{-0.01}^{+0.01}$ & $2.24_{-0.03}^{+0.03}$ \\
& $k T_{\mathrm{e}}(\mathrm{keV})$ & $>257$ & $>98$ \\
& $k T_{\text {in }}(\mathrm{keV})^{\mathrm{f}}$ & tied & tied \\
& norm & $0.042_{-0.002}^{+0.002}$ & $0.019_{-0.002}^{+0.001}$ \\
\hline
\end{tabular}

Notes.

${ }^{a}$ Errors are reported at the $90 \%$ confidence level and calculated. There were no distinctions made for the emissivities for the coronal flavor models in the inner and outer disk, so they were fixed such that Index $_{1}=$ Index $_{2}=3$. The dimensionless spin parameter and redshift were set to zero for the RELXILLNS model.

$\mathrm{b} \dagger$ is fixed for all observations.

${ }^{c}$ A hard limit of $70^{\circ}$ was placed.

${ }^{\mathrm{d}}$ A hard limit of 0.5 was placed.

e A hard limit of 3 was placed, see Section 3.2.

f This value is tied with the $T_{\text {in }}$ value in DISKBB.

in half-width frequency implies an increase in inner radius by a factor of eight from the intermediate state to the hard state.

The relationship between coronal temperature, power-law index, and inner radius (summarized in Table 4) is best understood by models consisting of an inner optically thin corona and an outer optically thick radius. In the early stages of the outburst, we see that the power-law index decreases as the coronal temperature decreases. This may be because soft photons from a disk cool the corona, producing a softer power- 
Table 4

Fit Parameter Summary

\begin{tabular}{|c|c|c|c|c|c|c|c|c|}
\hline MJD & State & DISKBB norm & $k T_{\text {in }}(\mathrm{keV})$ & $k T_{\mathrm{e}}(\mathrm{keV})$ & $\bar{\Gamma}$ & $R_{\text {in }}(\mathrm{km})^{\mathrm{a}}$ & FWHM $/ 2^{\mathrm{b}}$ & rms (\%) \\
\hline 58,792 & soft & $\cdots$ & $\cdots$ & $>114$ & $2.09_{-0.01}^{+0.01}$ & $\cdots$ & $\cdots$ & $\cdots$ \\
\hline 58,801 & soft & $3964_{-378}^{+410}$ & $0.45_{-0.03}^{+0.02}$ & $>257$ & $2.06_{-0.01}^{+0.01}$ & $\left(84_{-26}^{+27}\right) D_{10}(\cos \theta)^{-1 / 2}$ & $\cdots$ & $\cdots$ \\
\hline 58,812 & soft & $3312_{-384}^{+319}$ & $0.44_{-0.01}^{+0.02}$ & $>98$ & $2.24_{-0.03}^{+0.03}$ & $\left(77_{-26}^{+24}\right) D_{10}(\cos \theta)^{-1 / 2}$ & $\cdots$ & $\ldots$ \\
\hline 58,866 & intermediate & $29689_{-5922}^{+7621}$ & $0.16_{-0.01}^{+0.01}$ & $>160$ & $1.85_{-0.01}^{+0.01}$ & $\left(229_{-116}^{+102}\right) D_{10}(\cos \theta)^{-1 / 2}$ & $0.64 \pm 0.08$ & $27 \pm 1$ \\
\hline 58,879 & hard & $\cdots$ & $\cdots$ & $25_{-5}^{+10}$ & $1.77_{-0.01}^{+0.01}$ & $\cdots$ & $0.10 \pm 0.01$ & $31 \pm 8$ \\
\hline 58,889 & hard & $\cdots$ & $\cdots$ & $>19$ & $1.79_{-0.01}^{+0.01}$ & $\cdots$ & $0.06 \pm 0.01$ & $32 \pm 2$ \\
\hline 58,915 & hard & $\cdots$ & $\cdots$ & $11_{-2}^{+6}$ & $1.82_{-0.03}^{+0.03}$ & $\cdots$ & $0.03 \pm 0.01$ & $33 \pm 3$ \\
\hline 58,964 & hard & $\cdots$ & $\cdots$ & $>5$ & $1.74_{-0.10}^{+0.10}$ & $\cdots$ & $\cdots$ & $\cdots$ \\
\hline
\end{tabular}

Notes.

${ }^{\mathrm{a}} D_{10}$ is the source distance in units of $10 \mathrm{kpc}$ and $\theta$ is the inclination angle.

b The half-width of the zero-centered Lorentzian in hertz.

law index. However, as the mass accretion rate drops, the inner disk radius moves away from the compact object, and the corona is subject to a lower flux of soft photons from the disk. This lower influx of soft photons causes the coronal spectrum to harden. The correlation between a decreasing half-width frequency and increasing power-law photon index, previously identified in the hard states of other black hole systems (Gilfanov et al. 1999; Revnivtsev et al. 2001; Kalemci 2002; Tomsick et al. 2004), is also seen in this outburst for MAXI J0637-430.

\subsection{Nature of the Compact Object of MAXI J0637-430}

We can make further inferences on MAXI J0637-430 by deriving the mass and distance of the compact object from the inner disk radius. For a nonrotating compact object, we derive a mass of $M=(9 \pm 3) D_{10}(\cos \theta)^{-1 / 2} M_{\odot}$ from the assumption that the ISCO lies at $6 R_{\mathrm{g}}$ for the nonrotating Schwarzschild black hole. However, as the spin is unconstrained in our spectral analysis, we also consider the possibility that the compact object of MAXI J0637-430 may be rotating and that the effects of spin are not evident in our spectra. In the extremal Kerr case, theoretical support and decades of empirical evidence motivate linking $R_{\mathrm{ISCO}}$ to $R_{\text {in }}$ (Steiner et al. 2011). The mass could therefore be up to six times larger than for the Schwarzschild assumption. Although we discuss the source distance further below, the lower limit of $6.5 \mathrm{kpc}$ derived by Jana et al. (2021) corresponds to $M>6.0 \pm 2.0 M_{\odot}$, which would require the presence of a black hole. The least massive black hole found so far has $M=3.3 M_{\odot}$ (Thompson et al. 2019), while neutron stars reach an upper range between 1.5 and $3 M_{\odot}$.

Although the extremal Kerr case still qualifies MAXI J0637-430 as a stellar black hole when we consider mass alone as an indicator, it is necessary to consider the implication it would have for distance. For a nonrotating black hole, we estimate distance by solving a system of two equations between the mass-distance relation derived in Section 3.2 and a fraction of the state-transition luminosity (Jana et al. 2021). To obtain the state-transition luminosity, we extrapolated the flux between XRT observations 00012172060 and 00012172064 , obtaining $1.15 \times 10^{-9} \mathrm{erg} \mathrm{cm}^{-2} \mathrm{~s}^{-1}$ for $0.5-10 \mathrm{keV}$, and multiplied it by $4 \pi D_{10}^{2}$. In a thorough study, Maccarone (2003) demonstrated that the state-transition luminosity for an LMXB typically falls between 0.01 and 0.04 of the Eddington limit, which is given by $L_{\mathrm{e}}=1.3 \times 10^{38}\left(M / M_{\odot}\right) \mathrm{erg} \mathrm{s}^{-1}$.
Solving

$$
\begin{aligned}
& 1.15 \times 10^{-9} \mathrm{erg} \mathrm{cm}^{-2} \mathrm{~s}^{-1} \times 4 \pi D_{10}^{2} \\
& =0.01 \times 1.3 \times 10^{38}\left(M / M_{\odot}\right) \mathrm{erg} \mathrm{s}^{-1}
\end{aligned}
$$

with the mass-distance Equation (2) derived from diskbb gives a distance between 5.9 and $11.5 \mathrm{kpc}$ for an inclination of $\theta=0$.

In the extremal Kerr scenario, our mass-distance equation is $M_{\text {in }}=(54 \pm 18) D_{10}(\cos \theta)^{-1 / 2} M_{\odot}$. This would imply a distance of at least $33 \mathrm{kpc}$. We consider that MAXI J0637-430 is at a Galactic latitude of $-20^{\circ}$. This would place the compact object at least $11 \mathrm{kpc}$ from the Galactic disk. As the Galactic disk is the birthplace of supergiant and massive stars that evolve into neutron stars or black holes, this would be unusual indeed. If the disk inclination is at the measured average of $\theta=64^{\circ}$, or if the state-transition luminosity is higher than 0.01 of the Eddington limit, it could qualify as a Galactic halo object. A nonrotating or low-spin case is more likely than this rare scenario.

It would be remiss not to consider the possibility of a neutron star as a compact object. Looking at the equation $R_{\text {in }}=(81 \pm 26) D_{10}(\cos \theta)^{-1 / 2} \mathrm{~km}$ in the soft state, taking a fiducial neutron star radius of $10 \mathrm{~km}$, and liberally setting $\theta=0$, the distance would be $2.0 \mathrm{kpc}$. This close range seems highly improbable considering the extremely low luminosity detected in the soft state, implying a $0.9 \%$ Eddington luminosity for a $1.4 M_{\odot}$ NS. This unlikely scenario seems to favor the BHXRB interpretation.

We draw comparisons to a study by Cackett et al. (2009) on reflection from the inner accretion disk around neutron stars. The disk-blackbody norm for nine neutron stars was found to be of the order of 100-200, with a high inner disk temperature of the order of 1 or $2 \mathrm{keV}$. MAXI J0637-430 does not mimic this consistent pattern of a notably hotter disk with a smaller inner radius, and instead exhibits a cool disk as described by Remillard \& McClintock (2006). The magnitudes of the inner disk normalization and temperature are comparable to the results of a returning radiation fit for BHXRB XTE J1550-564 (Connors et al. 2020).

\subsection{Broadband Spectrum and Concluding Remarks}

While previous studies found that the soft states of the MAXI J0637-430 outburst are well described by a twocomponent model, NuSTAR's broader bandpass at $3-79 \mathrm{keV}$ improves the quality of the spectrum and introduces positive 


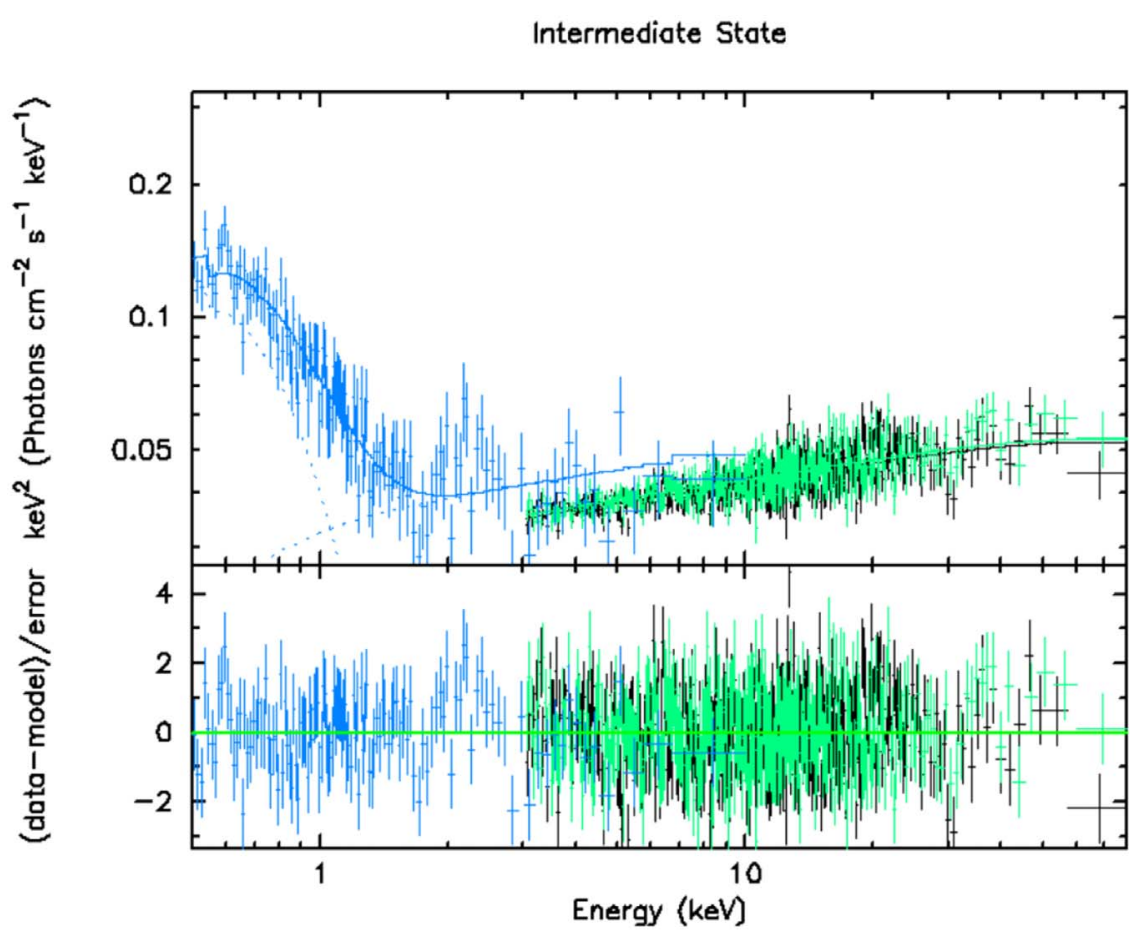

Figure 6. Spectra for NuSTAR's 80502324008 observation and XRT's 00012172066 observation fitted with a disk blackbody and a thermal Comptonization component.

residuals in the iron line region and above $10 \mathrm{keV}$. We tested both excess emission from the plunging region and two reflection scenarios. We conclude that excess emission from the plunging region yields a physically plausible width of a thin ring at the ISCO. However, we found that including a returning radiation reflection component gives the best fits, while also providing physically plausible estimates for the inner edge of the disk and mass of the black hole.

In testing reflection components, we found that the spectra of MAXI J0637-430 favor the softer, blackbody-like spectrum of the relxillNS model over the power-law-like coronal IC spectrum of the relxillcp model. The addition of this feature causes some changes worth noting. As an extra emission component is accounted for in the relxillNS model, it drops the temperature of the inner disk blackbody when compared to models that omit reflection (as seen by comparing to the $0.5-10 \mathrm{keV}$ analysis by Tetarenko et al. 2020). Although the residuals near the iron line were originally interpreted as iron emission features (Tomsick et al. 2019), they were not detected in this analysis. This may be due to the low iron abundance $\left(A_{\mathrm{Fe}}\right)$ derived from our spectral fits, which give a best fit at the minimum value used in creating the relxillNS model ( 0.5 solar). This analysis demonstrates that a reflection component describes the spectra well despite nondetection of the iron line.

It is interesting to note that typically halo stars originating from globular clusters have lower metallicities than stars in the Galactic plane, so a black hole accreting material from a halo companion star would be expected to have a low iron abundance. Regardless of this indicator, MAXI J0637-430 as a Galactic halo object would still be unusual. If MAXI J0637-430 has a black hole as a compact object, a massive star progenitor would be required, which is rare in the halo.

To gain a further understanding of accretion efficiency, or the fraction of energy actually radiated of that available according to the mass transfer rate into the accretion disk, we can calculate the ratio of our observed luminosity to the Eddington limit. Taking the high source flux on MJD 58,801 for $0.5-10 \mathrm{keV}$ and assuming a source distance of $8.7 \mathrm{kpc}$, we find $L_{\mathrm{obs}} / L_{\mathrm{e}}=0.029$, a relatively low value for a soft state (Maccarone 2003; Motlagh et al. 2019). The ionization parameter found by relxillns, which is low in the context of a BHXRB, can explain this low fraction of observed luminosity.

Though the reflection spectrum of relxillNS gives the best fits, we emphasize that in the soft state we find that the ratio of the flux of the reflection component to the flux of the disk-blackbody component is $\sim 0.6$ on average. Cunningham (1976) demonstrates that a returning radiation component is prominent for objects with spins between 0.9 and 0.998 , and thus this flux ratio may cast doubt on the nonrotating scenario. But as explained in the section above, a high spin would imply an unusually large distance from the Galactic disk. This disconnect presents a challenge to the scenario of returning radiation in this case and motivates further studies on the prominence of returning radiation flux for spins below 0.9 .

We have proposed two possible models for the soft-state X-ray spectrum of MAXI J0637-430, i.e., emission from the plunging region or reprocessing of returning disk radiation. Although the present study cannot confirm or rule out either of these two models, perhaps the most important conclusion is that the broadband spectra in the soft state are not adequately described by a two-component model based on the "standard" power-law continuum and associated reflection spectrum, such as in previous studies. The complexities observed in our data are not evident in a more limited energy bandwidth. This situation is not unique to MAXI J0637-430, but has been reported in studies of other sources in their soft state (e.g., MAXI J1820+070, Fabian et al. 2020; XTE J1550-564, Connors et al. 2020; EXO 1846-031, Wang et al. 2021; BHB 4U 1630-47 outburst, Connors et al. 2021). 


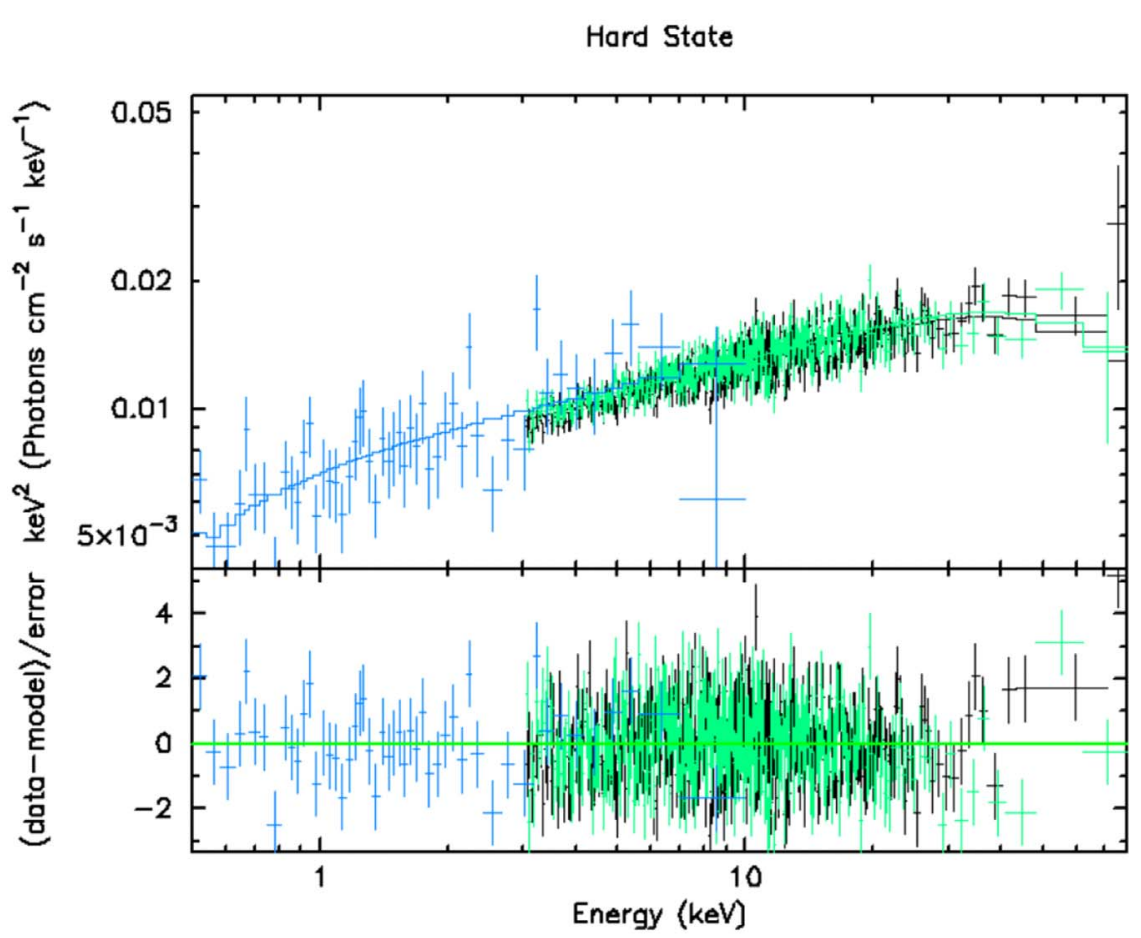

Figure 7. Spectra for NuSTAR's 80502324010 observation and XRT's 00012172077 observation fitted with a thermal Comptonization component.

Moreover, the validity of one model does not necessarily rule out the other, because it is possible that the most suitable model is a combination of both scenarios-the plunging region and returning radiation. The plunging region resides between the ISCO and event horizon, and thus its emission diminishes as the ISCO nears the event horizon; such is the case as maximal spin is approached. It is also feasible to postulate that some photons from the inner disk are bent back by the strong gravity of the black hole and reflected off the disk surface, but the fraction of photons experiencing this phenomenon depends strongly on the spin and geometry of illumination. A high fraction of returning photons is expected to occur at high spin. As the measurements throughout the outburst gave unconstrained values for spin, we derived the extremal cases of the mass-distance equation based on assumptions of the location of the ISCO, which depends on the extremal spin cases. Setting limits on distance with quiescent measurements could in turn better constrain the spin of the system. We therefore recommend quiescent optical and near-IR coverage data to be collected in order to better determine the spin of the system, which would help in determining the contribution of different model components. This will also help in evaluating the rare possibility of a distant halo object in addition to developing a model. Nonetheless, the options presented here are valuable when putting together a picture of MAXI J0637-430, because the broadband spectra require complex models that invoke physics of the inner accretion disk close to the complex object.

This work made use of data from the NuSTAR mission, a project led by the California Institute of Technology, managed by the Jet Propulsion Laboratory, and funded by the National Aeronautics and Space Administration. We thank the NuSTAR Operations, Software and Calibration teams for support with the execution and analysis of these observations. This research has made use of the NuSTAR Data Analysis Software
(NuSTARDAS) jointly developed by the ASI Science Data Center (ASDC, Italy) and the California Institute of Technology (USA). This work also made use of data from the Neil Gehrels Swift Observatory. H.L. and J.A.T. acknowledge partial support under NASA NuSTAR Guest Investigator grant 80NSSC20K0644. J.H. acknowledges support from an appointment to the NASA Postdoctoral Program at the Goddard Space Flight Center, administered by the Universities Space Research Association under contract with NASA. J.A.G. acknowledges support from NASA NuSTAR Guest Investigator grant 80NSSC20K1238, and from the Alexander von Humboldt Foundation. J.J. acknowledges support from the fellowship of China Postdoctoral Science Foundation (2021M691822), the Tsinghua Shuimu Scholar Program and the Tsinghua Astrophysics Outstanding Fellowship. Thank you to Dr. Thomas Dauser and Dr. Jack Steiner for the useful comments along the way.

Facilities: NuSTAR, Swift/XRT.

Software: XSPEC (v12.11.1c Arnaud 1996), HEAsoft (v6.27.2), relxill suite (Dauser et al. 2014; García et al. 2014).

\section{Appendix}

Every model tested produces positive residuals at energies below $1 \mathrm{keV}$ (see Figures 3-5). The optical spectroscopy analysis of Tetarenko et al. (2020) carefully derived the hydrogen column density from Ultraviolet and Optical Telescope measurements of MAXI J0637-430, so we fixed it to the hydrogen column density of $4.39 \times 10^{20} \mathrm{~cm}^{-2}$. Similarly, HI4PI ${ }^{13}$ find a Galactic column density in the direction of the source of $5.23 \times 10^{20} \mathrm{~cm}^{-2}$. However, without knowing the distance to the source, it could be argued that the estimates of global dust or column density of neutral atomic hydrogen determined by HI4PI can only serve as an upper bound.

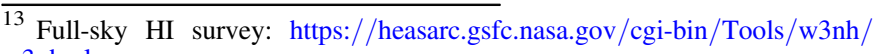
w3nh.pl.
} 
The Evolution of MAXI J0637-430
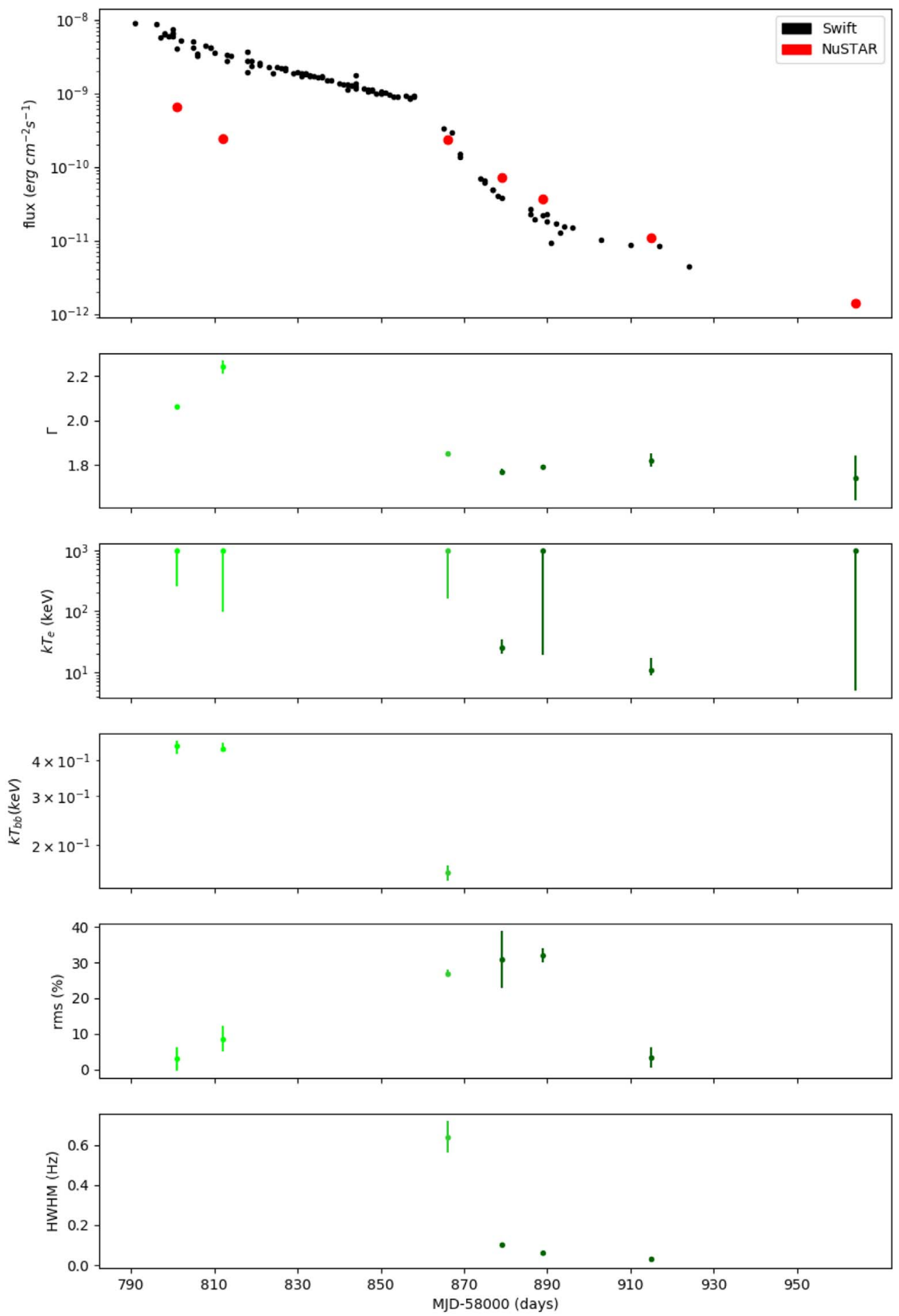

Figure 8. The evolution of (top to bottom) (a) the source flux for XRT (0.5-10 keV) (Tetarenko et al. 2020) and NuSTAR (3-79 keV), (b) the power-law photon index, (c) the electron temperature in $\mathrm{keV}$, (d) the inner disk temperature in $\mathrm{keV}$, (e) the rms (\%) derived from the power spectra, and (f) break frequencies from power spectra.

One option for the lower-energy residuals could be variation in elemental abundances in the interstellar medium. To test this, we fit to the model tbnew_feo $x$ (diskbb + relxillNS + Nthcomp), in which tbnew_feo from the absmodel ${ }^{14}$ package is a form of the Tbabs component that considers

$\overline{14}$ https://pulsar.sternwarte.uni-erlangen.de/wilms/research/tbabs/ variation in oxygen and iron abundances. Taking the column density to be $4.39 \times 10^{20} \mathrm{~cm}^{-2}$, and tying the oxygen and iron abundances together, we find that the reduced $\chi^{2}$ shows only slight improvement for MJD 58,801 at 825 for 714 dof, with the $<1 \mathrm{keV}$ residuals slightly smaller but still present. The two elemental abundances fit to a subsolar value of $<0.6$ solar. 


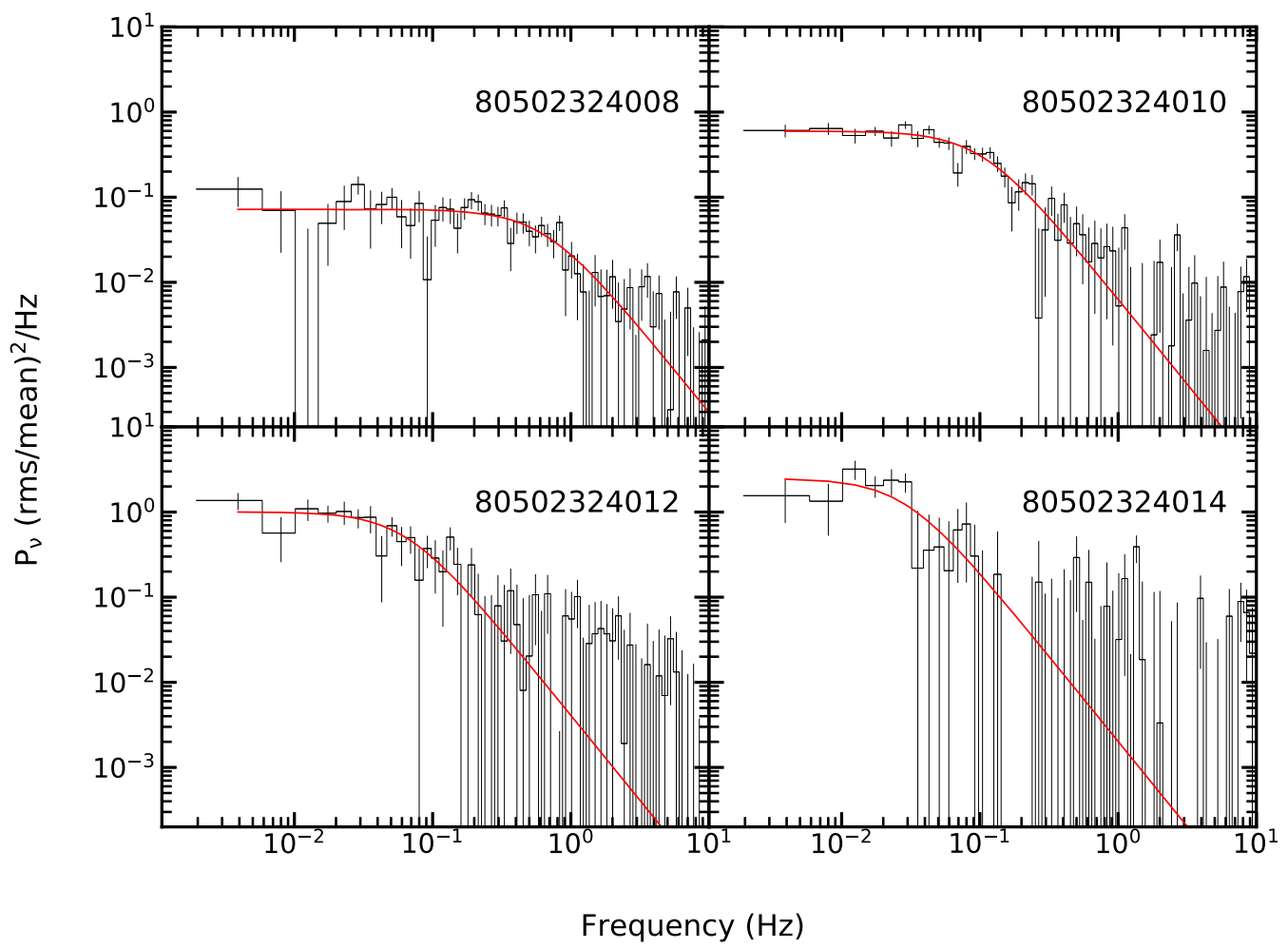

Figure 9. The average cospectra for FPMA and FPMB for hard and soft-hard NuSTAR observations (MJD 58,866, 58,879, 58,889, and 58,915).

Soft State: Reduced Column Density

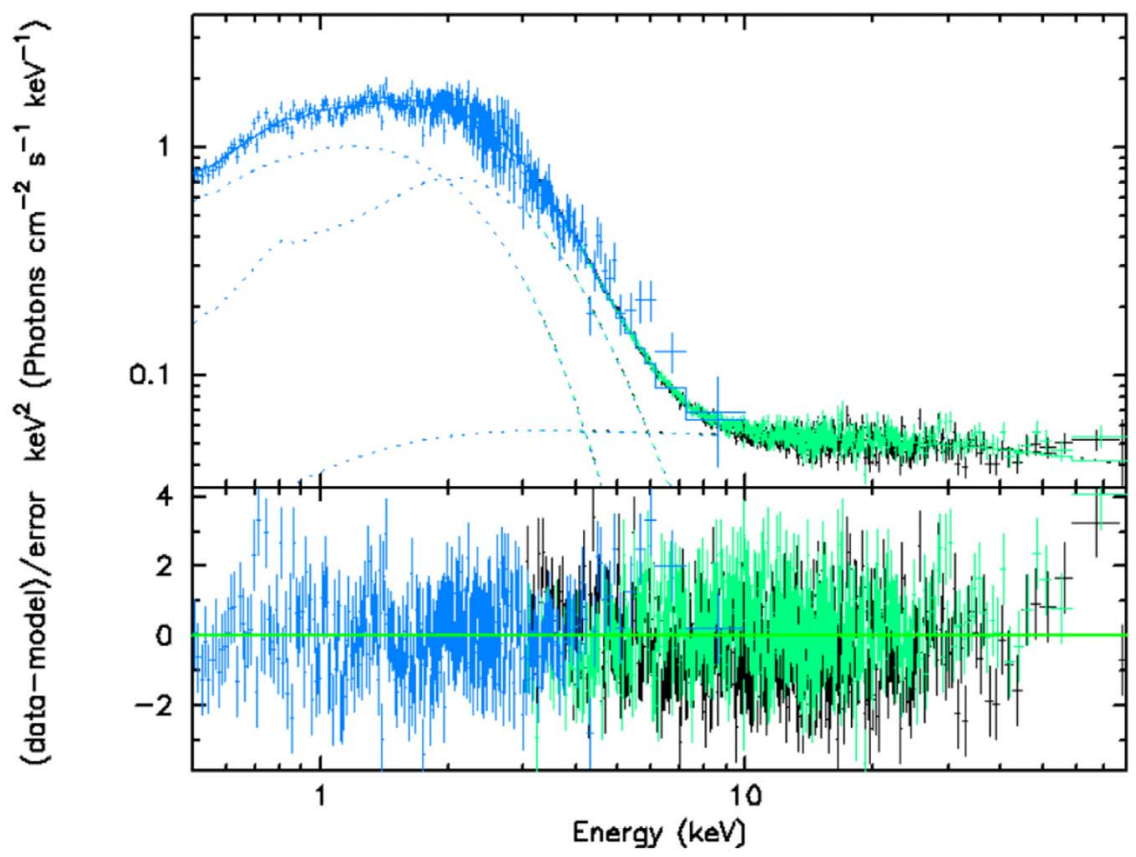

Figure 10. Spectra for NuSTAR's 80502324004 observation and XRT's 00012172008 observation fitted with components for a disk blackbody, thermal Comptonization, and reflection of blackbody returning radiation. Column density converged to $2.26 \times 10^{20} \mathrm{~cm}^{-2}$.

To check whether a lower value of the column density eliminates the low-energy residuals, we repeated the analysis for the soft state and found the column density from the fit to the XRT and NuSTAR spectra. For the most statistically robust observation (MJD 58,801), the returning radiation model gave that the column density is equal to $(2 \pm 1) \times 10^{20} \mathrm{~cm}^{-2}$, and the plunging region model gave that the column density is
$3_{-2}^{+4} \times 10^{20} \mathrm{~cm}^{-2}$. The $\chi^{2}$ value remained about the same for the plunging region model at 2158 for 1156 dof, and improved slightly for the returning radiation model at $\chi^{2}$ of 1311 for 1151 dof. The residuals below $<1 \mathrm{keV}$ were diminished (Figure 10). We note that the change in column density did not alter the parameter values outside the error ranges listed in Table 3 and for the $R_{\text {in }}$ values in Table 4 . 
Table 5

Reflection/Disk-blackbody Flux Ratio in the Soft State

\begin{tabular}{lcc}
\hline \hline$N_{\mathrm{H}}\left(\mathrm{cm}^{-2}\right)$ & MJD 58,801 & MJD 58,812 \\
\hline $4.39 \times 10^{20}$ & 0.8 & 0.4 \\
$(1.47-2.62) \times 10^{20}$ & 0.7 & 0.3 \\
\hline
\end{tabular}

The change in column density slightly reduces the ratio of the flux of the reflection component and the flux of the diskblackbody component, summarized in Table 5. For a column density either fixed to $4.39 \times 10^{20} \mathrm{~cm}^{-2}$ or found from the fitted spectra, the ratio is notably high for the earlier observation on MJD 58,801. If the column density is $4.39 \times 10^{20} \mathrm{~cm}^{-2}$ or higher, then there may be an extra emission component in the early stages of the soft state currently unaccounted for in all models.

\section{ORCID iDs}

Hadar Lazar (1D https://orcid.org/0000-0002-2752-9524

John A. Tomsick (10 https://orcid.org/0000-0001-5506-9855

Sean N. Pike (1) https://orcid.org/0000-0002-8403-0041

Matteo Bachetti (i) https://orcid.org/0000-0002-4576-9337

Riley M. T. Connors (iD https://orcid.org/0000-00028908-759X

Andrew C. Fabian (iD https://orcid.org/0000-0002-9378-4072

Felix Fuerst (ib https://orcid.org/0000-0003-0388-0560

Javier A. García (10) https://orcid.org/0000-0003-3828-2448

Jeremy Hare (iD https://orcid.org/0000-0002-8548-482X

Aarran W. Shaw (i) https://orcid.org/0000-0002-8808-520X

Dominic J. Walton (i) https://orcid.org/0000-0001-5819-3552

\section{References}

Arnaud, K. A. 1996, in ASP Conf. Ser. 101, Astronomical Data Analysis Software and Systems V, ed. G. H. Jacoby \& J Barnes (San Francisco, CA: ASP), 17

Bachetti, M., Harrison, F. A., Cook, R., et al. 2015, ApJ, 800, 109

Balbus, S. A., \& Hawley, J. F. 1991, ApJ, 376, 214
Burrows, D. N., Hill, J. E., Nousek, J. A., et al. 2005, SSRv, 120, 165 Cackett, E. M., Miller, J. M., \& Ballantyne, D. R. 2009, ApJ, 720, 205 Connors, R. M. T., Garcia, J., Dauser, T., et al. 2020, ApJ, 892, 47 Connors, R. M. T., García, J., Tomsick, J. A., et al. 2021, ApJ, 909, 146 Cunningham, C. 1976, ApJ, 208, 534

Dauser, T., García, J., Parker, M. L., Fabian, A. C., \& Wilms, J. 2014 MNRAS, 444, L100

Di Matteo, T., \& Psaltis, D. 1999, ApJ, 526, L101

Dubus, G., Hameury, J.-M., \& Lasota, J.-P. 2001, A\&A, 373, 251

Fabian, A., Buisson, D. J., Kosec, P., et al. 2020, MNRAS, 493, 5389

Fabian, A. C., Rees, M. J., Stella, L., \& White, N. E. 1989, MNRAS, 238, 729

García, J., Dauser, T., Lohfink, A., et al. 2014, ApJ, 782, 76

Gilfanov, M., Churazov, E., \& Revnivtsev, M. 1999, A\&A, 352, 182

Haardt, F., \& Maraschi, L. 1993, ApJ, 413, 507

Harrison, F. A., Craig, W. W., Christensen, F. E., et al. 2013, ApJ, 770, 103

Huppenkothen, D., Bachetti, M., Stevens, A. L., et al. 2019, ApJ, 881, 39

Jana, A., Jaisawal, G. K., Naik, S., et al. 2021, MNRAS, 504, 4793

Kalemci, E. 2002, PhD Thesis, Univ. California at San Diego

Kubota, A., Tanaka, Y., Makishima, K., et al. 1998, PASJ, 50, 667

Lasota, J. P. 2001, NewAR, 45, 449

Liu, Q. Z., van Paradijs, J., \& van den Heuvel, E. P. J. 2007, A\&A, 469, 807 Maccarone, T. J. 2003, A\&A, 409, 697

Madsen, K., Harrison, F. A., Markwardt, C. B., et al. 2015, ApJS, 220, 8

Makishima, K., Maejima, Y., Mitsuda, K., et al. 1986, ApJ, 308, 635

Mitsuda, K., Inoue, H., Koyama, K., et al. 1984, PASJ, 36, 741

Motlagh, A. V., Kalemci, E., \& Maccarone, T. J. 2019, MNRAS, 485, 2744

Negoro, H., Miike, K., Nakajima, W., et al. 2019, ATel, 13256, 1

Novikov, I. D., \& Thorne, K. S. 1973, in Black Holes (Les Astres Occlus), ed.

C. Dewitt \& B. S. Dewitt (London: Gordon and Breach), 343

Remillard, R. A., \& McClintock, J. E. 2006, ARA\&A, 44, 49

Revnivtsev, M., Gilfanov, M., \& Churazov, E. 2001, A\&A, 380, 520

Shakura, N. I., \& Sunyaev, R. A. 1973, A\&A, 500, 33

Shimura, T., \& Takahara, F. 1995, ApJ, 445, 780

Steiner, J. F., Reis, R. C., McClintock, J. E., et al. 2011, MNRAS, 416, 941

Strader, J., Aydi, E., Sokolovsky, K., et al. 2019, ATel, 13260, 1

Tetarenko, B. E., Lasota, J. P., Heinke, C., et al. 2018, Natur, 554, 69

Tetarenko, B. E., Shaw, A. W., Manrow, E. R., et al. 2020, MNRAS, 501, 3406

Thompson, A. T., Kochanek, C. S., Stanek, K. Z., et al. 2019, Sci, 366, 637

Tomsick, J. A., García, J., Fabian, A., et al. 2019, ATel, 13270

Tomsick, J. A., Kalemci, E., \& Kaaret, P. 2004, ApJ, 601, 439

Tomsick, J. A., \& Lazar, H. 2020, ATel, 13800, 1

Wang, Y., Long, J., García, J., et al. 2021, ApJ, 542, 914

Wilms, J., Allen, A., \& McCray, R. 2000, ApJ, 542, 914

Zdziarski, A. A., Johnson, W. N., \& Magdziarz, P. 1996, MNRAS, 283, 193

Zdziarski, A. A., Zycki, P. T., Svensson, R., \& Boldt, E. 1993, ApJ, 405, 125 